\title{
El impacto de la crisis sanitaria COVID-19 en la administración de las organizaciones de Economía Popular y Solidaria
}

\section{The impact of the COVID-19 health crisis on the administration of the Popular and Solidarity Economy organizations}

\author{
David Armando León Pasquel $^{1 *}$, Josselyn Gabriel Ayuy Dutan ${ }^{1}$, María Magdalena Tsukanka Sharup ${ }^{1}$ \\ ${ }^{1}$ Universidad Católica de Cuenca, Ecuador \\ *geli1996zabala@gmail.com
}

DOI: https://doi.org/10.26871/killkanasocial.v5i1.699

\begin{abstract}
Resumen
El presente trabajo analiza el impacto de la crisis sanitaria Covid-19 en la administración de las organizaciones de Economía Popular y Solidaria de las Provincias de Pastaza y Morona Santiago del sector agropecuario mediante una investigación a las organizaciones pertenecientes a IEPS y de igual forma a las entidades que brindan apoyo a este sector productivo, de esta forma se obtendrá información necesaria para la realización del presente trabajo educativo. Esto es necesario en base de que las organizaciones y empresas a nivel mundial han experimentado inconvenientes en sus actividades comerciales, generando de este modo inconvenientes económicos tanto de manera asociativa como individual de cada socio y trabajador. La investigación en sí, se centra en la relación existente entre las asociaciones agropecuarias y los organismos gubernamentales, específicamente con el Instituto de Economía Popular y Solidaría quienes han establecido conexiones fuertes con los emprendimientos asociativos en las provincias de todo el país. La investigación, es de carácter exploratorio, en donde se establece un estudio de la situación actual que está viviendo la economía del país por los impactos que el Covid-19 ha generado en los últimos meses, utilizando para su medición la recolección de datos de diversas fuentes, entre ellas encuestas mediante las cuales se procesa información de las asociaciones pertinentes, siendo estas mismas encuestadas posteriormente para conocer con veracidad el impacto de la crisis sanitaria covid-19 en la administración de las organizaciones de economía popular y solidaria de las provincias de Pastaza y Morona Santiago del sector agropecuario. Dichos datos expresan que las asociaciones de interés han sufrido grandes impactos tanto administrativos, como de distribución de productos y adquisición de materia prima puesto que la política gubernamental establecida a favor del cuidado de la ciudadanía frente a la crisis sanitaría ocasionaron cambios drásticos en la actividad comercial cotidiana hasta el momento. Se puede concluir en el estudio que existen diversos efectos debido a la crisis sanitaria del COVID-19, entre los cuales destacan los procesos administrativos de las organizaciones de Economía Popular y Solidaria en las provincias de Morona Santiago y Pastaza, puesto que, si bien hubo colaboración del Estado en algunos aspectos, esta entidad no pudo cubrir adecuadamente con los requerimientos de las organizaciones a las que se debe.
\end{abstract}

Palabras clave: COVID-19, Crisis, IEPS, procesos administrativos, productividad, sector agropecuario.

\begin{abstract}
The present work analyzes the impact of the Covid-19 health crisis on the administration of the organizations of Popular and Solidarity Economy of the Provinces of Pastaza and Morona Santiago in the agricultural sector through an investigation to the organizations belonging to IEPS and in the same way to the entities that provide support to this productive sector, thus the necessary information is required to carry out this educational work. This is necessary in the base of the organizations and companies worldwide have experienced inconveniences in their commercial activities, thus generating economic inconveniences both associatively and individually for each partner and worker. The research itself focuses on the relationship between agricultural associations and government agencies, specifically with the Institute of Popular and Solidarity Economy, which have established strong connections with associative ventures in provinces across the country. The research is exploratory in nature, establishing a study of the current situation that the country's economy is experiencing due to the impacts that the Covid-19 has generated in recent months, using data collection from various measurements for its measurement. sources, including evaluations through which information from the relevant associations is processed, these same subsequent surveys being used to know with truth the impact of the covid-19 health crisis on the administration of organizations of popular and solidarity economy in the provinces of Pastaza and Morona Santiago from the agricultural production sector. These data express that the associations of interest have suffered great administrative impacts, as well as the distribution of products and the acquisition of raw materials since the government policy established in favor of caring for citizens in the face of the health crisis caused drastic changes in activity. everyday commercial so far. It can be concluded in the study that there are various effects due to the health crisis of COVID-19, which include the administrative processes of the organizations of Popular and Solidarity Economy in the provinces of Morona Santiago and Pastaza, since, although there was collaboration of the State in some aspects, this entity could not meet the requirements of the organizations to which it is due.
\end{abstract}

Keywords: Administrative processes, Agricultural sector, COVID-19, crisis, IEPS, productivity. 


\section{Introducción}

Con el presente trabajo se pretende evaluar el impacto de la crisis sanitaria COVID-19 en los procesos administrativos de las organizaciones de Economía Popular y Solidaria provincias de Pastaza y Morona Santiago del sector agropecuario mediante una investigación a las organizaciones pertenecientes a IEPS y de igual forma a las entidades que brindan apoyo a este sector productivo, de esta forma se obtendrá información necesaria para la realización del presente trabajo educativo.

Esto por medio del cumplimiento de los siguientes objetivos, que permitirán conocer los efectos de la crisis sanitaria COVID-19 en los procesos administrativos de las organizaciones de Economía Popular y Solidaria en las provincias de Morona Santiago y Pastaza a través de medios periodísticos y datos proporcionados por más organizaciones afectadas para el conocimiento acertado de la situación que se está atravesando esta parte de la economía del país; identificar los mecanismos de intercambio comercial de las organizaciones de Economía Popular y Solidaria en las provincias de Morona Santiago y Pastaza mediante entrevistas a los involucrados en estos procesos productivos, así se conocerá de qué forma se comercializa dentro y fuera de la provincia y analizar la relación existente entre los procesos administrativos y los mecanismos de intercambio comercial de las organizaciones de Economía Popular y Solidaria las provincias de Morona Santiago y Pastaza por medio de una evaluación de los resultados obtenidos dentro de la investigación de forma que se realice un análisis claro y competente.

Así, este trabajo se centra en el sector agropecuario, que es aquel trabajo relacionado tanto como por la agricultura como por la ganadería, el cual pertenece al sector primario de producción (González, 2008); actualmente este se enfrenta a la globalización, por ende, es importante trabajar en ello mediante información científica e innovación tecnológica que permita solventar proyectos sostenibles (Gutiérrez, Calle, y Agudelo, 2018).

La agricultura dentro de la economía amazónica cumple un papel fundamental puesto que la mayoría de su población se dedica a trabajos enfocados en dicha área (Houtart, 2018), a su vez, existen diversas organizaciones que se dedican a fomentar este sector, entre ellos destaca la Superintendencia de Economía Popular y Solidaria (SEPS), de la que muchos productores forman parte, la cual busca fomentar la solidaridad, cooperación y reciprocidad (Houtart, 2014).

El 11 de marzo del 2020 la OMS (Organización Mundial de la Salud), declara al covid-19 como una pandemia, tras esto la mayoría de países han entrado en una crisis sanitaria, humana y económica sin precedentes en el último siglo (Serra, 2020). Esto ha afectado notoriamente al sector productivo, generando dificultades económicas a numerosas familias que dependen tanto directa como indirectamente de este, frente a esto en muchos sectores del país se han intentado generar diversas alternativas con las cuales se pueda estabilizar en la medida de lo posible dicha situación (Albertí y cols., s.f.).

Actualmente la economía ecuatoriana se está enfrentando a unos de los peores escenarios de la historia, comenzando con la caída del petróleo, y con el covid-2019, ya que este virus está ocasionando fuertes estragos a los ciudadanos (Terranova, Masacón, y Ulloa, 2020), la mayoría han dejado de trabajar e intentan retomar su normalidad con nuevas normativas incorporadas, las empresas pequeñas están decayendo, el turismo está drásticamente afectado, y el sector manufacturero está completamente congelado (De la Luz Bajaña Mendieta, 2020).

Por su parte el Instituto de Economía Popular y Solidaria (IEPS), el cual busca participar directamente en la transformación de la matriz productiva, aportando estrategias al desarrollo socioeconómico del país (Torres, N., E., y Alonso Alemán, 2017) cuenta con diferentes mecanismos que están poniendo a disposición de las asociaciones (de Economía Popular y Solidario., 2013) entre ellas las agropecuarias para fomentar la subsistencia durante la crisis sanitaria, a su vez, existen otras organizaciones que buscan ayudar a este sector de la economía ya que se ha visto muy afectado por no poder desempeñar con normalidad sus actividades (por los Derechos Humanos, 2020).

Es importante recalcar que una de las instituciones que está brindando un apoyo al sector agropecuario es el Ministerio de Agricultura y Ganadería (MAG) la cual ha realizado la recolección de productos que provienen de asociaciones agrícolas locales para la venta ayudando así a la economía de los agricultores en función de que puedan continuar con sus actividades con la máxima normalidad posible.

Pese al trabajo que han realizado diversos organismos gubernamentales, es palpable el malestar económico que se está dando en Ecuador (Rojo, 2020), el cual más allá de cumplir con la estadística propuesta ha resultado en un impacto mucho más fuerte, que a su vez ha derivado en una crisis social sin precedente, es por ello que es necesario conocer la realidad y trabajar desde ese punto (Prendes y Herrera, 2020).

De este modo, se podrá clarificar para quienes hagan uso de este documento posteriormente la importancia que tiene el sector agropecuario dentro de la economía del país y que este, debe ser más explotado e impulsado por el estado, está claro que la crisis sanitaria por la que está atravesando el país hoy en día es una baja muy grande para la administración de este tipo de organizaciones y por ello es importante segregar la información para obtener y detallar los puntos más importantes que existen y detallar las medidas que se están tomando por parte de las entidades e instituciones reguladoras para contrarrestar de cierta forma los problemas por los que está atravesando el sector agropecuario. 


\section{Metodología}

\subsection{Modelo de investigación}

El presente trabajo es de carácter exploratorio donde se establece un estudio de la situación actual que está viviendo la economía del país por los impactos que el Covid-19 ha generado en los últimos meses, para esto se utilizó como herramienta la recolección de datos de diversas fuentes entre ellas encuestas mediante las cuales se procesó información de las asociaciones pertinentes, siendo estas encuestadas posteriormente para conocer con veracidad el impacto de la crisis sanitaria covid-19 en la administración de las organizaciones de economía popular y solidaria de las provincias de Pastaza y morona Santiago del sector de producción agropecuaria.

Se obtuvo una base de datos general, en donde las asociaciones agropecuarias de estudio, ascendían a 58, de las cuales se procedió a tomar una muestra para el trabajo.

Debido a que el tamaño del universo es inferior a 100, y que la situación actual impide la encuesta en persona, el único modo de obtener información es mediante llamadas telefónicas, por lo que se ha procedido a realizar una muestra aleatoria, está en base a los datos proporcionados por el Instituto de Economía Popular y Solidaria (IEPS), correspondiendo a 26 en la Provincia de Morona Santiago y 32 en la Provincia de Pastaza.

\section{Resultados}

\subsection{Asociaciones de estudio}

De las asociaciones generadas por la base de datos general, aquellas que se dedican al área agropecuaria las Provincias de estudio dan como resultado 58, de las cuales, se han encuestado un total de 20 asociaciones, encontrándose 8 en la Provincia de Morona Santiago, presentando un 40,00\% del total, mientras que los 12 restantes se encuentran en la Provincia de Pastaza, siendo representada por un $60,00 \%$.

\subsubsection{Porcentaje en el que ha sido afectado el emprendi- miento debido a la crisis sanitaria mundial}

Debido a la crisis sanitaria mundial la economía se ha visto gravemente afectada, siendo así también para los emprendimientos, en el caso de estudio, se ha llegado a constatar por medio de los socios que en la mayoría de asociaciones agropecuarias se han visto fuertemente dañadas, estableciendo un porcentaje de daño que fluctúa entre el $75 \%$ al $100 \%$ como porcentajes generales, puesto que estos son los más destacados por los afectados, dado que reiteran haber sufrido contratiempos de diversa índole, tanto administrativos, como de operación, y de recursos humanos, así como el inconveniente de adquirir materia prima u obtener compradores en el transcurso de tiempo que duró el cierre de negocios y toque de queda a nivel nacional, con lo cual se generaron problemas económicos tanto personalmente como a nivel empresarial, pues en este período se suscitaron grandes pérdidas por la mayoría de asociaciones.

\subsubsection{Impacto en las funciones administrativas del em- prendimiento en la crisis sanitaria del Covid-19}

La crisis sanitaría que se ha vivido de manera global debida a la contaminación masiva del Covid-19, ha tenido diversos impactos en los emprendimientos, en el caso de estudio, las asociaciones han recalcado que se han visto fuertemente afectadas de manera administrativa, impidiendo la planificación, organización, dirección y control de forma normal, ocasionando grandes inconvenientes a la asociación en general.

Según las asociaciones encuestadas, la mayoría han sido fuertemente afectadas administrativamente, pues han sufrido de diversas complicaciones, que en la mayoría de los casos aún no han sido cubiertos por completo, pues requieren de tiempo, dinero y poner en marcha muchas operaciones que han estado estancadas; por otra parte, otras asociaciones consideran haber tenido daños moderados, y se están recuperando poco a poco, teniendo complicaciones pero pudiendo contrarrestarlas y seguir adelante; del mismo modo, existen menos asociaciones que han sido levemente afectadas, las cuales han tenido dificultades dentro de lo que pueden manejar y están reaccionando de manera adecuada a la reactivación de su emprendimiento; y por último y en menor número existen asociaciones que si bien consideran ha habido cambios en el entorno que han afectado a la administración directa como indirectamente no se sienten específicamente afectados, pues pueden seguir con el emprendimiento sin mayores complicaciones.

\subsubsection{Aspectos en los que la crisis sanitaria (COVID-19) ha afectado al emprendimiento}

La crisis sanitaría, ha afectado a los emprendimientos de múltiples maneras, sin embargo, cabe resaltar que lo más representativo es la falta de pagos de clientes a tiempo debido a problemas económicos; en segundo lugar, el problema más destacable es la ausencia de trabajadores en sus puestos de trabajo, ya sea por enfermedad o por cuidado de los niños, en este aspecto el gobierno tuvo gran parte de responsabilidad, pues debido a la restricción vehicular y cierre de trabajos parcialmente los empleados no pudieron cumplir con sus obligaciones de manera adecuada; en tercer lugar, las asociaciones agropecuarias debido a la crisis sanitaria se han visto en la obligación de reducir los servicios de logística; paralelamente existen asociaciones que debido a la crisis sanitaria han tenido problemas con la infraestructura, ya sean con el internet, el transporte, u otros; del mismo modo, pero en menor grado las asociaciones han expresado que en algunos casos se han visto obligadas a cerrar temporalmente, otras han visto mayores cuellos de botellas administrativos, mientras que diversas de ellas han tenido reducción de inversiones y finalmente una minoría no consideran haber sido afectados directamente por la crisis sanitaria. 


\subsubsection{La crisis sanitaria (COVID-19) y su efecto en la capacidad de compra y/o venta de insumos en las empresas}

La crisis sanitaria debida al Covid-19 ha afectado la capacidad de las empresas de diversas maneras, entre las cuales destaca el haber experimentado menores ventas locales a consumidores, así como menores ventas locales a empresas, esto se debe principalmente a que los consumidores finales han disminuido sus compras debido a una crisis económica familiar, la cual ha sido expandida a la vez que se ha presentado diversos inconvenientes de movilización tanto de manera comercial como de forma individual a civiles.

Del mismo modo, pero en menor cantidad existen asociaciones agropecuarias que consideran haber experimentado dificultades para acceder a insumos de manera local debido a la crisis sanitaria; por último, una mínima parte de empresas han experimentado otros inconvenientes comerciales debido a la crisis sanitaria.

\subsubsection{Implementación de nuevas estrategias de marketing y comercialización para llegar a los consumidores con productos o servicios}

Debido a la crisis sanitaria que se ha dado debido al Covid-19 y su rápido contagio, se han dado múltiples inconvenientes económicos debido a que han existido falencias en el sistema comercial convencional, es por ello que la mayoría de asociaciones se han visto en la necesidad de implementar nuevas estrategias de marketing y comercialización para llegar a los consumidores con sus productos y servicios, si bien esto no ha sido en todos los casos, sí ha sido una cifra elevada de empresas quienes han optado por estas medidas.

\subsubsection{Estrategias por las que optaron los emprendimien- tos para hacer frente a la crisis de COVID 19}

Debido a la crisis sanitaria, las asociaciones se vieron en la necesidad de poner en marcha ciertas estrategias para hacer frente a dicha situación, en tal caso, la mayor parte de ellas, optaron por reducir temporalmente el empleo o despedir empleados; por otra parte, también se optó por incrementar sus esfuerzos en marketing o implementar el teletrabajo; a la vez, otras estrategias variaban entre implementar ventas en línea o implementar productos nuevos o personalizados.

Por último, una mínima parte de las empresas reprogramaron sus préstamos bancarios o tomaron otras alternativas.

\subsubsection{Principales inconvenientes a los que se enfren- tan los emprendimientos como consecuencia del COVID-19}

Como consecuencia de la emergencia sanitaria del Covid-19, los emprendimientos se enfrentan a diversos problemas, entre los que sobresale el no poder trabajar de manera normal por enfermedad u órdenes del gobierno, pues este último emitió decretos que reducían el comercio y la movilización nacional con el fin de salvaguardar la salud de los ciudadanos.

Por otro lado, un gran inconveniente al que se enfrentaron los emprendimientos en este periodo de tiempo y que aún suscita cierto malestar es la insuficiencia de flujo de efectivo para mantener el personal y las operaciones comerciales, puesto que varias de las empresas han tenido que reducir el número de trabajadores temporalmente y en casos más extremos han tenido que despedirlos.

Del mismo modo, se ha notado un inconveniente para proveerse de materia prima, interrumpiendo por esta razón las operaciones productivas, reduciendo así la posibilidad de comercialización; paralelamente, también los clientes han sido afectados disminuyendo la demanda.

También, los proveedores han experimentado problemas en cuanto al suministro de los insumos necesarios en el periodo de tiempo establecido, esto se debe en gran parte a los decretos establecidos por el Estado.

Todos estos inconvenientes, han generado malestar con las asociaciones, por los que las mismas han reiterado que no han operado con normalidad y han interrumpido sus procesos, debido a la situación a la que se han estado enfrentado.

\section{Análisis}

Las asociaciones totales de estudio son 20, de las cuales se encuentran equitativamente distribuidas en Morona Santiago y Pastaza, con 8 y 12 respectivamente; todas ellas han sido conscientes de los cambios ocasionados debido a la emergencia sanitaria, pues la economía se ha visto gravemente afectada, siendo así también para los emprendimientos, sin embargo, su porcentaje de afección es variable, siendo la mayoría afectado entre $50 \%$ y $75 \%$; esto a su vez, ha tenido un impacto que oscila entre lo moderado y fuerte en el área administrativa, puesto que se han visto más complicaciones de las normales.

Al ser fuertemente afectadas de manera administrativa, se ha impedido la planificación, organización, dirección y control de forma normal, ocasionando grandes inconvenientes a la asociación en general, siendo así que en la mayoría de los casos aún no han sido cubiertos por completo, pues requieren de tiempo, dinero y poner en marcha muchas operaciones que han estado estancadas.

Los principales problemas a los que los emprendimientos han sido expuestos, han sido la ausencia de los empleados y a la reducción de inversiones, así como clientes que no han pagado a tiempo sus facturas, a su vez, la crisis sanitaria ha afectado la capacidad de compra y/o venta de productos en todos sus aspectos. Debido a esto, más de las tres cuartas partes de las asociaciones han implementado nuevas estrategias de marketing y comercialización para llegar a los consumidores con sus productos o servicios frente a la crisis sanitaria del Covid-19. 


\section{Conclusiones}

Se puede concluir en el presente estudio que existen diversos efectos debido a la crisis sanitaria del COVID19, entre los cuales destacan los procesos administrativos de las organizaciones de Economía Popular y Solidaria en las provincias de Morona Santiago y Pastaza, puesto que, si bien hubo colaboración del Estado en algunos aspectos, esta entidad no pudo cubrir adecuadamente con los requerimientos de las organizaciones a las que se debe.

Se ha podido constatar que ha existido un intercambio comercial a cargo de las organizaciones de Economía Popular y Solidaria en las provincias de Morona Santiago y Pastaza, sin embargo, este no ha sido en favor de todas las asociaciones, puesto que únicamente se realizó esta actividad en asociaciones dedicadas a la manufactura textil, comercializando con centros tanto públicos como privados; sin embargo, sí se ha llevado a cabo una interacción económica con agricultores, sin embargo estas han sido a cargo de MAG y no a asociaciones como tal sino de manera individual a individuos o comerciantes para generar apoyo ante esta emergencia.

Por último, debido a que la cooperación a las organizaciones de Economía Popular y Solidaria no abarcó a aquellas dedicadas al área agropecuaria, los procesos de intercambio no fueron realizados mediante este sistema, por lo que las asociaciones individualmente tuvieron que tomar medidas de contingencia en relación a los procesos administrativos, los cuales si bien se han visto afectados drásticamente, en la mayoría de casos tomarán varios meses para recuperarse y poder continuar con sus actividades de manera normal.

\section{Recomendaciones}

Se ha de considerar que las asociaciones merecen apoyo por parte de entidades gubernamentales que faciliten el desarrollo de su actividad, finalidad que aún en situaciones de emergencia pues si bien su comercio fue frenado, su producto pudo haber sido despachado o intercambiado de diversas formas fomentando la economía y evitando que el trabajo de los más vulnerables sea violentado en épocas tan difíciles.

\section{Referencias Bibliográficas}

Albertí, A., Bageneta, M., Bardomás, S., Bober, G., Lombardi, J., Mott, M. M., y Scirica, S. (s.f.). Los trabajadores temporarios de la agricultura frente al covid-19.

de Economía Popular y Solidario., I. N. (2013). La economía popular y solidaria el ser humano sobre el capital 2007-2013.

De la Luz Bajaña Mendieta, I. (2020). Incidencias del covid-19 en ecuador. Questión.

González, A. N. (2008). El sector agropecuario en cuba. Nueva sociedad, 216, 77-89.

Gutiérrez, L., Calle, C., y Agudelo, G. (2018). Política de transferencia tecnológica del sector agropecuario colombiano con enfoque territorial. Lecturas de Economía, 89, 199-219.

Houtart, F. (2014). El desafío de la agricultura campesina para el ecuador. la restauración conservadora del correísmo. , 167-178.

Houtart, F. (2018). La agricultura campesina e indígena como una transición hacia el bien común de la humanidad: el caso de ecuador. Desacatos, 56, 177-187.

por los Derechos Humanos, A. (2020). Intervención humanitaria de la onu por covid-19. Descargado de http://repositorio.dpe.gob.ec/ handle/39000/2459

Prendes, N. M., y Herrera, M. L. M. (2020). Impacto social de la covid-19 en brasil y ecuador: donde la realidad supera las estadísticas. EDUMECENTRO, 12(3), 277-283.

Terranova, K. T. T., Masacón, N. . H., y Ulloa, W. L. O. (2020). Impacto del covid-19 en la planeación estratégica de las pymes ecuatorianas. RECIMUNDO, 4(3), 76-85.

Torres, P., N., F. L., E., P., y Alonso Alemán, A. (2017). Balance de la economía popular y solidaria en ecuador. Economía y Desarrollo, 158(1), 180-196.

Recibido: 26 de febrero de 2021

Aceptado: 20 de marzo de 2021 
